Validation Study

\title{
Single-Laboratory Validation Study of Rapid Analysis Method for Multi-Class Veterinary Drugs in Milk, Fish and Shellfish by LC-MS/MS
}

(Received March 1, 2013)

\author{
Takayuki Nakajima*, Chieko Nagano, Maki Kanda, Hiroshi Hayashi, Tsuneo Hashimoto, \\ Setsuko Kanai, Yoko Matsushima, Yukinari Tateishi, \\ Takeo SASAmoto and Ichiro TAKano
}

Tokyo Metropolitan Institute of Public Health: 3-24-1 Hyakunin-cho, Shinjuku-ku, Tokyo 169-0073, Japan; * Corresponding author

\begin{abstract}
A method of rapid analysis of multi-class residual veterinary drugs in milk, fish and shellfish was validated in accordance with Japanese guidelines for the validation of analytical methods for residual agricultural chemicals in food. Using LC-MS/MS, 43 multi-class veterinary drugs, including sulfonamides, quinolones, coccidiostats and antiparasites, could be analyzed in one injection. Analytes were extracted from samples with two kinds of solvent, acetonitrile containing 1 vol\% formic acid and anhydrous acetonitrile, and salted out with $4.0 \mathrm{~g}$ of magnesium sulfate, $1.5 \mathrm{~g}$ of trisodium citrate and $2.0 \mathrm{~g}$ of sodium chloride. This method was assessed by performing recovery tests in retail milk and 4 kinds of fresh cultured fish and shellfish (salmon, tiger shrimp, red sea bream and bastard halibut) spiked with the 43 target analytes at the levels of 10 and $100 \mu \mathrm{g} / \mathrm{kg}$. Using this method, 40 out of 43 drugs satisfied the guideline criteria in milk, 37 drugs in salmon, 42 drugs in tiger shrimp, 41 drugs in red sea bream and 39 drugs in bastard halibut.
\end{abstract}

Key words: veterinary drug, milk; fish and shellfish; LC-MS/MS, multi-class analysis; singlelaboratory validation study

\section{Introduction}

Veterinary drugs are used worldwide for treatment and prevention of animal diseases, and as food additives for growth promotion. However, some of those drugs can remain and accumulate in animal muscle due to overadministration or noncompliance with withdrawal period guidelines. Residual veterinary drugs pose a potential risk of allergic reactions, pathogen resistance and cytotoxicity in humans ${ }^{1)}$; therefore, it is important to monitor foods for residual veterinary drugs.

There are two major analytical strategies for the analysis of residual substances, namely, multi-class analysis $^{2-7)}$ and individual analysis of each compound ${ }^{8)-10)}$. However, quick and easy multi-class analysis has become more and more attractive because of the development of sophisticated analytic equipment, the diversification of medicines used, and increasing governmental requests for the analysis of foods available on the market. In addition, the analytic methodologies used by the inspection institutes have to be validated by 13/12/2013 in line with a notice issued by the Ministry of Health, Labour and Welfare of Japan. Therefore, a simple, rapid and simultaneous analysis of veterinary drugs is highly

* Takayuki_1_Nakajima@member.metro.tokyo.jp desirable.

However, there are some issues that have yet to be resolved in order to validate the simultaneous analysis of veterinary drugs:

1) A wide variety of drugs must be simultaneously extracted, ranging from hydrophilic compounds, such as antibiotics and fluoroquinolones, to lipophilic compounds, such as macrolide antiparasites and polyether antibiotics.

2) The exact drugs that are detected in both domestic and foreign inspection institutes have to be confirmed.

3) Many consumable supplies such as reagents, solvents, SPEs and HPLC columns are required.

4) It is hard to secure sufficient time and human resources to perform validation study, and it is difficult to guarantee the precision of the analytical method, especially inter-day repeatability, if the extraction process is complicated.

We developed a simple, rapid and simultaneous analytical method for residual veterinary drugs that covered the inspection items of the Tokyo Metropolitan Institute of Public Health by modifying the QuEChERS methodol$\mathrm{ogy}^{11)}$. In that previous study, the new methodology was successfully applied and validated for bovine muscle, swine muscle, chicken muscle and avian eggs. Therefore, 
in this study, our multi-class analysis was applied to a wider range of food samples and validated in accordance with the Japanese guidelines $* 1$

\section{Materials and Methods}

\section{Chemicals and Reagents}

Powdered standards for testing residual veterinary drug grades were used: ciprofloxacin hydrochloride (purity: 98.0\%), clopidol (98.7\%), closantel sodium (99.0\%), decoquinate $(97.0 \%)$, fenbendazole $(98.0 \%)$, florfenicol (98.0\%), 5-hydroxythiabendazole (98.0\%), oxfendazole (98.0\%), oxfendazole sulfone (99.9\%), sulfadimethoxine (99.0\%), sulfamethoxazole (99.0\%), triclabendazole (99.0\%), triclabendazole sulfone (98.0\%), triclabendazole sulfoxide (98.0\%) and trimethoprim (99.0\%) were purchased from Wako Pure Chemical Industries, Ltd. (Osaka, Japan). Danofloxacin mesylate (99.5\%), enrofloxacin (99.8\%), levamisole hydrochloride (99.0\%), nalidixic acid (99.8\%), nicarbazin (99.8\%), oxolinic acid (98.8\%), pyrimethamine (99.8\%), sulfadimidine (99.4\%), sulfamerazine $(99.5 \%)$, sulfathiazole $(99.9 \%)$, sulfaquinoxaline sodium (99.6\%) and thiabendazole (99.0\%) were purchased from Kanto Chemical Co. (Tokyo, Japan). Diclazuril (99.8\%), eprinomectin (99.3\%), flubendazole (99.9\%) and 5-propylsulfonyl-1 $H$-benzimidazole-2-amine (99.1\%) were purchased from Hayashi Pure Chemical Industries (Osaka, Japan). Doramectin (94.8\%), monensin sodium (86.3\%), moxidectin (97.1\%), narasin $(97.0 \%)$ semduramicin sodium $(96.1 \%)$, salinomycin $(93.8 \%)$, sarafloxacin hydrochloride (97.3\%) and $100 \mu \mathrm{g} / \mathrm{mL}$ solution of lasalocid A sodium were purchased from Sigma-Aldrich (St. Louis, MO, USA). Ivermectin (96.0\%), maduramicin ammonium (85.5\%) and ormethoprim (99.0\%) were purchased from Dr. Ehrenstorfer GmbH (Augsburg, Germany).

A stock standard solution of danofloxacin was prepared as below: transfer about $5 \mathrm{mg}$ of powdered standard, accurately weighed, to a $50 \mathrm{~mL}$ of round-bottom flask, and then dilute with methanol to the concentration of $100 \mu \mathrm{g} / \mathrm{mL}$. A closantel solution was prepared by dissolving about $5 \mathrm{mg}$ of powder, accurately weighed, in $5 \mathrm{~mL}$ of tetrahydrofuran, and diluting the resulting solution with methanol to the concentration of $100 \mu \mathrm{g} / \mathrm{mL}$. Other analytes were prepared in accordance with the method outlined in the previous study ${ }^{11}$. These solutions were stored at $4^{\circ} \mathrm{C}$ for up to one year. A working standard solution was prepared by mixing $500 \mu \mathrm{L}$ of all stock standard solutions and diluting with acetonitrile to $50 \mathrm{~mL}$. This solution was stored at $4^{\circ} \mathrm{C}$ for up to three months.

Acetonitrile (HPLC grade), formic acid (LC/MS grade), ammonium acetate (LC/MS grade), magnesium sulfate (analytical grade), trisodium citrate dehydrate (analytical grade) and sodium chloride (analytical grade) were

${ }^{* 1}$ Guidelines for the Validation of Analytical Method for Residual Agricultural Chemicals in Food (December 24, 2010, Notification 1224, No. 1). The Ministry of Health, Labour and Welfare of Japan purchased from Wako Pure Chemical Industries. Methanol (HPLC grade), tetrahydrofuran (analytical grade) and dimethylformamide (analytical grade) were purchased from Kanto Chemical Co. Ultrapure water was obtained from a Milli-Q water system (Merck Millipore, Billerica, MA, USA).

\section{Samples}

Retail milk and 4 kinds of fresh cultured fish and shellfish (salmon, tiger shrimp, red sea bream and bastard halibut) were purchased from local supermarkets in Tokyo and were confirmed to be free of the targeted analytes. The tissue samples were minced with an electric food processor and stored at $-20^{\circ} \mathrm{C}$. Milk was stored at $4^{\circ} \mathrm{C}$.

\section{$L C-M S / M S$ conditions}

The LC-MS/MS conditions basically followed those of the previous study ${ }^{11)}$. Briefly, LC separation was performed using a Prominence 20A series (Shimadzu Corporation, Kyoto, Japan), and Syncronis aQ (2.1 mm i.d. $\times 100 \mathrm{~mm}, 5 \mu \mathrm{m}$, Thermo Fisher Scientific, Waltham, MA, USA) as an LC column at $40^{\circ} \mathrm{C}$. The gradient was constructed form $0.1 \%$ formic acid in $10 \mathrm{mmol} / \mathrm{L}$ ammonium acetate $(\mathrm{A})$ and acetonitrile $(\mathrm{B})$. The initial condition was $(\mathrm{A}):(\mathrm{B})=95: 5$. The acetonitrile concentration was increased to $95 \%$ for $7 \mathrm{~min}$, and this was maintained for $5 \mathrm{~min}$. Then, the mobile phases were re-equilibrated to the initial condition for $5 \mathrm{~min}$. The flow rate was $0.4 \mathrm{~mL} / \mathrm{min}$. The injection volume was $3 \mu \mathrm{L}$.

MS detection was performed using a TSQ Quantum Access MAX (Thermo Fisher Scientific), and positive and negative electrospray ionization with voltages of $3.0 \mathrm{kV}$ and $2.5 \mathrm{kV}$, respectively. The vaporizer temperature was $460^{\circ} \mathrm{C}$ and the capillary temperature was $220^{\circ} \mathrm{C}$. Optimization of MS/MS parameters was performed by direct infusion of $1 \mu \mathrm{g} / \mathrm{mL}$ of each standard solution, and the optimized conditions for selective reaction monitoring (SRM) transitions are shown in Table 1.

\section{Sample preparation}

Five grams of each sample was placed into a $50 \mathrm{~mL}$ polypropylene centrifuge tube, then $50 \mu \mathrm{L}$ or $500 \mu \mathrm{L}$ of working standard solution was added. After waiting for more than $30 \mathrm{~min}, 5 \mathrm{~mL}$ of water (except in the case of milk) and $15 \mathrm{~mL}$ of $1 \mathrm{vol} \%$ formic acid in acetonitrile (Method A) or acetonitrile alone (Method B) were added. Tissue samples were homogenized thoroughly and the milk was well shaken. Next, $4 \mathrm{~g}$ of magnesium sulfate, $1.5 \mathrm{~g}$ of trisodium citrate dehydrate and $2 \mathrm{~g}$ of sodium chloride were added to the tube, which was immediately shaken by hand for $1 \mathrm{~min}$. The mixture was centrifuged at $1,800 \times g$ for $10 \mathrm{~min}$, and the supernatant was transferred into a 20 -mL volumetric flask. After dilution to volume with extraction solvent, a portion of the solution was transferred to a $1.5 \mathrm{~mL}$ microtube and centrifuged at $16,000 \times g$ for $10 \mathrm{~min}$. Three $\mu \mathrm{L}$ of the supernatant was injected into the LC-MS/MS. 
Table 1. MS/MS parameters of each analyte

\begin{tabular}{|c|c|c|c|c|c|c|}
\hline \multirow[b]{2}{*}{ Analyte } & \multirow{2}{*}{$\begin{array}{l}\text { M.R.W. } \\
\quad(\min )\end{array}$} & \multicolumn{5}{|c|}{ SRM transition } \\
\hline & & ESI & $\begin{array}{l}\text { Precursor } \\
\text { ion }(m / z)\end{array}$ & $\begin{array}{l}\text { Product } \\
\text { ion }(m / z)\end{array}$ & $\begin{array}{c}\text { Collision } \\
\text { energy (eV) }\end{array}$ & $\begin{array}{l}\text { Tube lens } \\
\text { (V) }\end{array}$ \\
\hline Clopidol & $2.2-3.0$ & + & 192 & 101 & 24 & 122 \\
\hline 5-Hydroxythiabendazole & $2.4-3.1$ & + & 218 & 108 & 37 & 105 \\
\hline Levamisole & $2.5-3.3$ & + & 205 & 91 & 35 & 102 \\
\hline 5-Propylsulfonyl- $1 H$-benzimidazole-2-amine & $2.5-3.3$ & + & 240 & 133 & 30 & 82 \\
\hline Sulfathiazole & $2.5-3.3$ & + & 256 & 92 & 26 & 109 \\
\hline Trimethoprim & $2.7-3.5$ & + & 291 & 123 & 26 & 94 \\
\hline Sulfamerazine & $2.8-3.6$ & + & 265 & 92 & 29 & 93 \\
\hline Ciprofloxacin & $2.8-3.6$ & + & 332 & 314 & 20 & 119 \\
\hline Danofloxacin & $2.9-3.7$ & + & 358 & 82 & 35 & 133 \\
\hline Ormethoprim & $2.9-3.7$ & + & 275 & 123 & 21 & 107 \\
\hline Enrofloxacin & $3.0-3.9$ & + & 360 & 316 & 18 & 116 \\
\hline Thiabendazole & $3.0-3.9$ & + & 202 & 65 & 40 & 106 \\
\hline Sulfadimidine & $3.0-4.0$ & + & 279 & 186 & 16 & 93 \\
\hline Sarafloxacin & $3.4-4.1$ & + & 386 & 368 & 26 & 138 \\
\hline Sulfamonomethoxine & $3.4-4.1$ & + & 281 & 108 & 25 & 82 \\
\hline Sulfamethoxazole & $3.5-4.3$ & + & 254 & 92 & 25 & 96 \\
\hline Florfenicol & $3.5-4.3$ & - & 356 & 336 & 13 & -87 \\
\hline Oxfendazole & $3.6-4.3$ & + & 316 & 159 & 31 & 120 \\
\hline Pyrimethamine & $3.8-4.8$ & + & 249 & 177 & 28 & 114 \\
\hline Oxolinic acid & $3.8-4.8$ & + & 262 & 244 & 19 & 106 \\
\hline Sulfaquinoxaline & $3.9-4.7$ & + & 301 & 108 & 26 & 95 \\
\hline Sulfadimethoxine & $3.9-4.7$ & + & 311 & 156 & 20 & 96 \\
\hline Oxfendazole sulfone & $4.0-4.8$ & + & 332 & 300 & 22 & 118 \\
\hline Nalidixic acid & $4.2-5.4$ & + & 233 & 215 & 13 & 97 \\
\hline Flubendazole & $4.5-5.3$ & + & 314 & 123 & 34 & 123 \\
\hline Fenbendazole & $5.2-6.0$ & + & 300 & 268 & 19 & 103 \\
\hline Nicarbazin & $5.4-6.6$ & - & 301 & 137 & 16 & -65 \\
\hline Triclabendazole sulfoxide & $5.4-6.4$ & - & 373 & 181 & 46 & -78 \\
\hline Diclazuril & $5.9-6.6$ & - & 405 & 334 & 22 & -70 \\
\hline Triclabendazole sulfone & $6.0-6.7$ & - & 391 & 312 & 29 & -102 \\
\hline Triclabendazole & $6.3-7.1$ & + & 359 & 198 & 25 & 124 \\
\hline Decoquinate & $7.0-8.0$ & + & 418 & 204 & 41 & 107 \\
\hline Eprinomectin & $7.0-8.0$ & + & 915 & 112 & 55 & 113 \\
\hline Closantel & $7.5-8.2$ & - & 661 & 345 & 37 & 106 \\
\hline Semduramicin & $7.5-8.5$ & + & 891 & 630 & 22 & 126 \\
\hline Doramectin & $7.5-8.6$ & + & 917 & 593 & 10 & 138 \\
\hline Lasalocid & $7.5-8.7$ & + & 609 & 237 & 23 & 112 \\
\hline Moxidectin & $7.9-8.9$ & + & 641 & 499 & 12 & 113 \\
\hline Ivermectin & $8.2-8.9$ & + & 893 & 570 & 13 & 113 \\
\hline Maduramicin & $8.5-9.1$ & + & 935 & 630 & 21 & 132 \\
\hline Salinomycin & $8.5-9.2$ & + & 769 & 734 & 17 & 94 \\
\hline Monensin & $8.6-9.3$ & + & 689 & 461 & 23 & 119 \\
\hline Narasin & $8.7-9.7$ & + & 783 & 748 & 17 & 114 \\
\hline
\end{tabular}

* Monitoring retention window

Table 2. Comparison of matrix effects in each extracted solution derived from food samples

\begin{tabular}{|c|c|c|c|c|c|c|c|c|c|c|}
\hline \multirow{2}{*}{ Response ratio* } & \multicolumn{2}{|c|}{ Milk } & \multicolumn{2}{|c|}{ Salmon } & \multicolumn{2}{|c|}{ Tiger shrimp } & \multicolumn{2}{|c|}{ Red sea bream } & \multicolumn{2}{|c|}{ Bastard halibut } \\
\hline & $\mathrm{A}$ & $\mathrm{B}$ & $\mathrm{A}$ & $\mathrm{B}$ & $\mathrm{A}$ & $\mathrm{B}$ & $\mathrm{A}$ & $\mathrm{B}$ & $\mathrm{A}$ & B \\
\hline$>1.2$ & 0 & 2 & 3 & 2 & 1 & 1 & 3 & 6 & 3 & 2 \\
\hline $1.1-1.2$ & 4 & 4 & 5 & 3 & 3 & 2 & 2 & 12 & 6 & 3 \\
\hline $0.9-1.1$ & 36 & 35 & 34 & 36 & 34 & 35 & 35 & 25 & 31 & 29 \\
\hline $0.8-0.9$ & 3 & 2 & 1 & 1 & 4 & 2 & 1 & 0 & 2 & 8 \\
\hline$<0.8$ & 0 & 0 & 0 & 1 & 1 & 3 & 2 & 0 & 1 & 1 \\
\hline
\end{tabular}

* Peak area ratio of matrix-matched standard solution/extraction solvent standard solution

All solutions were fortified at $25 \mu \mathrm{g} / \mathrm{L}$ 


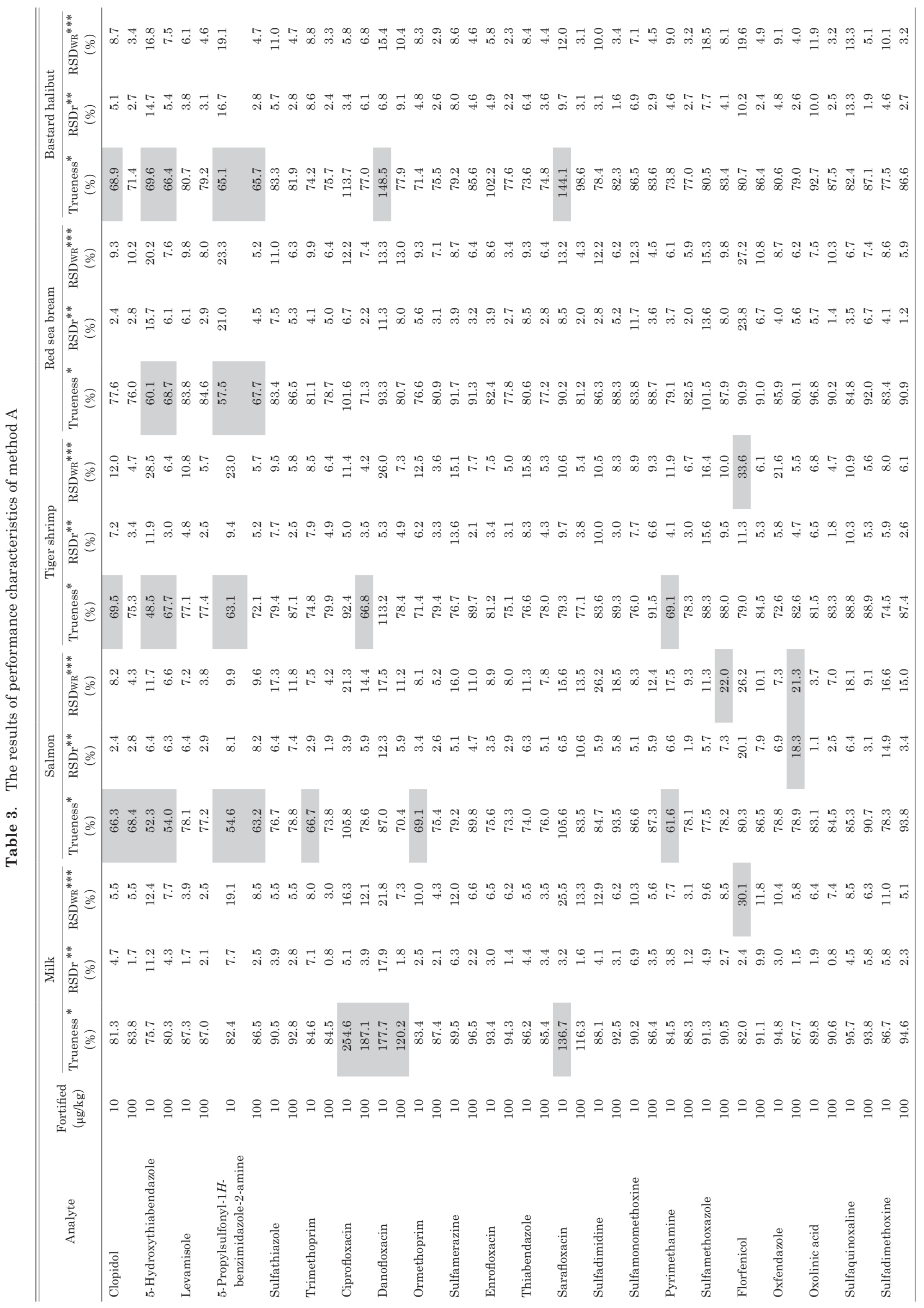




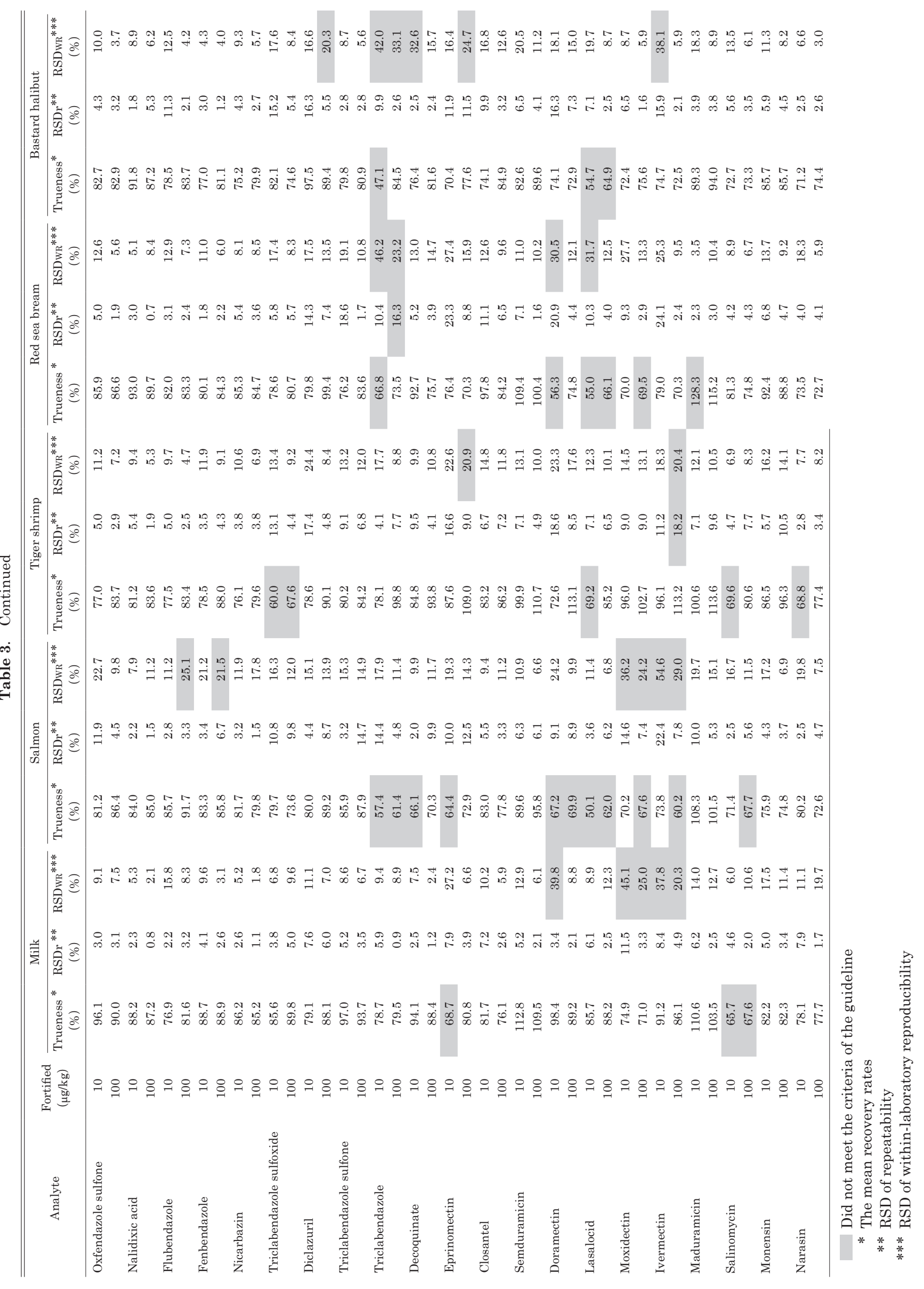







| क्: 象 * F |

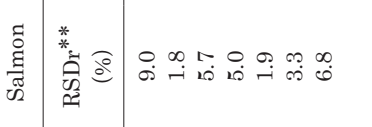

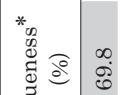

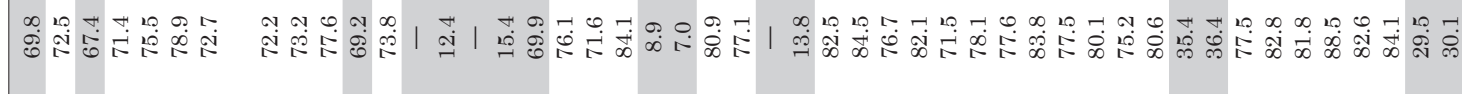
草 *

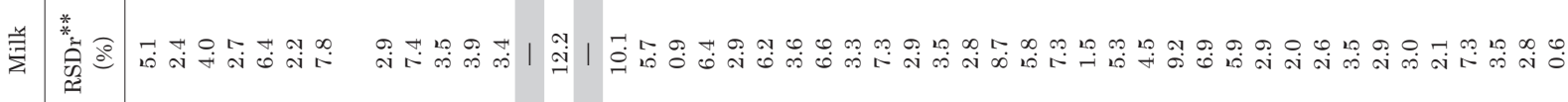
*

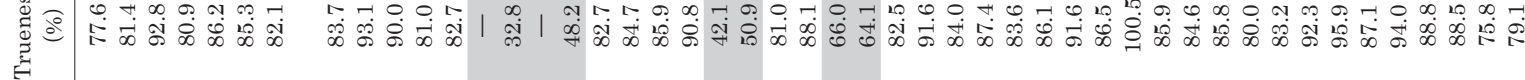
莺憵

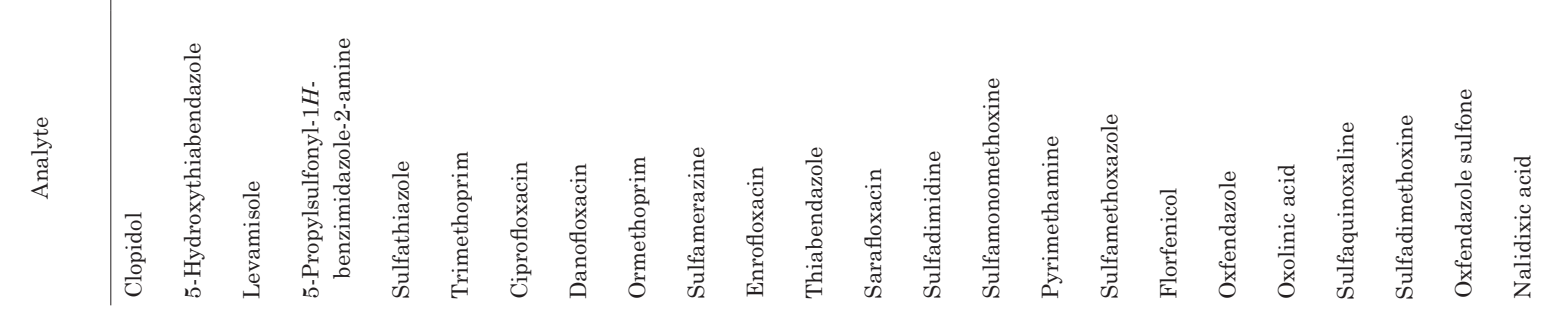




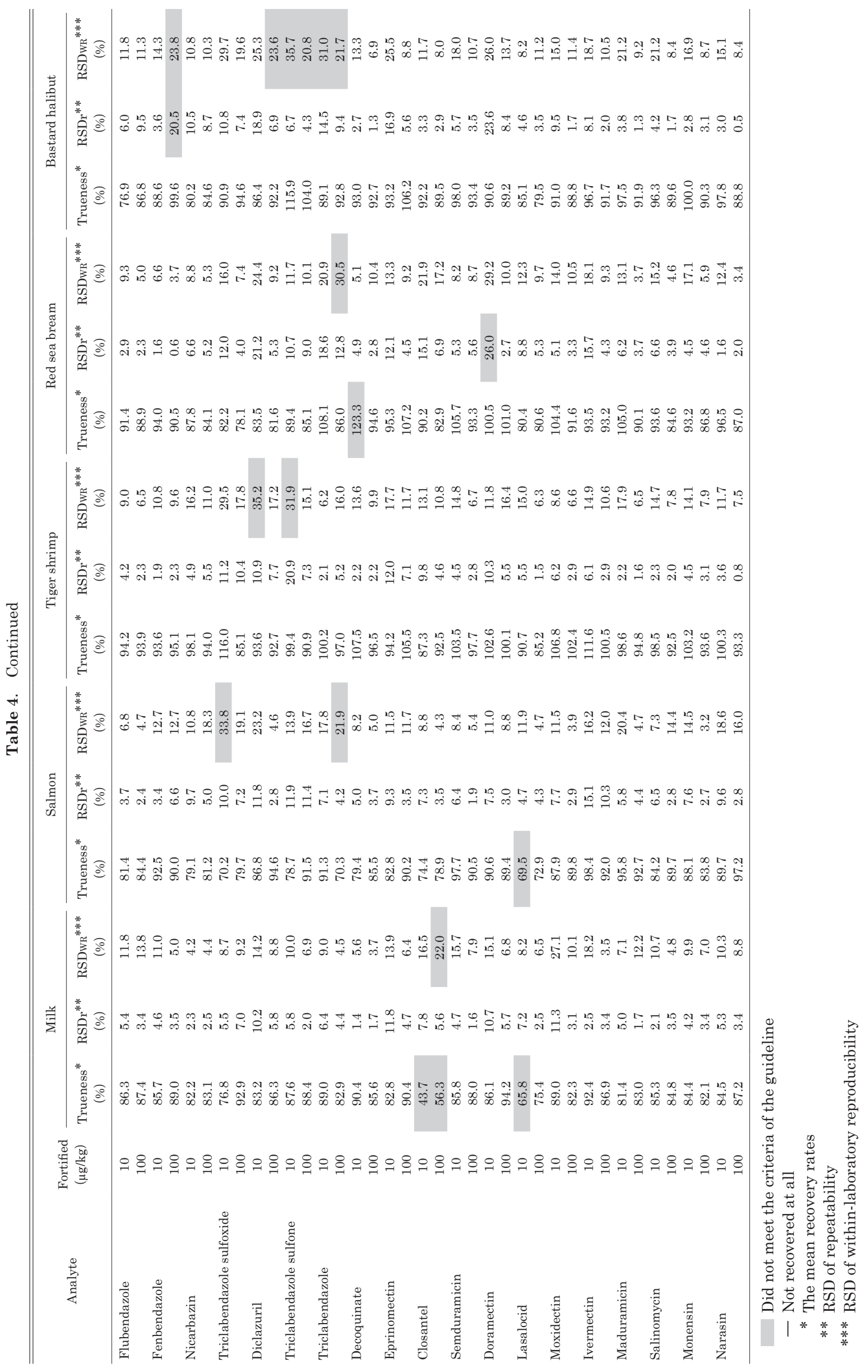


Table 5. Validated method and LOQ of each analyte

\begin{tabular}{|c|c|c|c|c|c|c|c|c|c|c|}
\hline \multirow[b]{2}{*}{ Analyte } & \multicolumn{2}{|c|}{ Milk } & \multicolumn{2}{|c|}{ Salmon } & \multicolumn{2}{|c|}{ Tiger shrimp } & \multicolumn{2}{|c|}{ Red sea bream } & \multicolumn{2}{|c|}{ Bastard halibut } \\
\hline & $\begin{array}{c}\text { Validated } \\
\text { method }\end{array}$ & $\begin{array}{c}\text { LOQ } \\
(\mu \mathrm{g} / \mathrm{kg})\end{array}$ & $\begin{array}{l}\text { Validated } \\
\text { method }\end{array}$ & $\begin{array}{c}\mathrm{LOQ} \\
(\mu \mathrm{g} / \mathrm{kg})\end{array}$ & $\begin{array}{l}\text { Validated } \\
\text { method }\end{array}$ & $\begin{array}{c}\text { LOQ } \\
(\mu \mathrm{g} / \mathrm{kg})\end{array}$ & $\begin{array}{l}\text { Validated } \\
\text { method }\end{array}$ & $\begin{array}{c}\text { LOQ } \\
(\mu \mathrm{g} / \mathrm{kg})\end{array}$ & $\begin{array}{l}\text { Validated } \\
\text { method }\end{array}$ & $\begin{array}{c}\text { LOQ } \\
(\mu \mathrm{g} / \mathrm{kg})\end{array}$ \\
\hline Clopidol & $\mathrm{A}, \mathrm{B}$ & 2 & - & - & B & 2 & $\mathrm{~A}, \mathrm{~B}$ & 4 & $\mathrm{~B}$ & 4 \\
\hline 5-Hydroxythiabendazole & A, B & 10 & - & - & B & 10 & $\mathrm{~B}$ & 10 & B & 10 \\
\hline Levamisole & A, B & 4 & $\mathrm{~A}, \mathrm{~B}$ & 4 & $\mathrm{~A}, \mathrm{~B}$ & 4 & $\mathrm{~A}, \mathrm{~B}$ & 4 & $\mathrm{~A}, \mathrm{~B}$ & 4 \\
\hline $\begin{array}{l}\text { 5-Propylsulfonyl- } 1 H \text { - } \\
\text { benzimidazole-2-amine }\end{array}$ & A, B & 2 & B & 2 & B & 2 & B & 4 & B & 2 \\
\hline Sulfathiazole & A, B & 1 & A, B & 1 & $\mathrm{~A}, \mathrm{~B}$ & 1 & A, B & 1 & $\mathrm{~A}, \mathrm{~B}$ & 1 \\
\hline Trimethoprim & $\mathrm{A}, \mathrm{B}$ & 1 & - & - & $\mathrm{A}, \mathrm{B}$ & 1 & $\mathrm{~A}, \mathrm{~B}$ & 1 & $\mathrm{~A}, \mathrm{~B}$ & 1 \\
\hline Ciprofloxacin & - & - & $\mathrm{A}$ & 10 & - & - & $\mathrm{A}$ & 4 & A & 10 \\
\hline Danofloxacin & - & - & $\mathrm{A}$ & 10 & $\mathrm{~A}$ & 10 & A & 10 & - & - \\
\hline Ormethoprim & $\mathrm{A}, \mathrm{B}$ & 1 & - & - & $\mathrm{A}, \mathrm{B}$ & 1 & $\mathrm{~A}, \mathrm{~B}$ & 1 & $\mathrm{~A}, \mathrm{~B}$ & 1 \\
\hline Sulfamerazine & $\mathrm{A}, \mathrm{B}$ & 1 & $\mathrm{~A}, \mathrm{~B}$ & 1 & $\mathrm{~A}, \mathrm{~B}$ & 1 & A, B & 1 & $\mathrm{~A}, \mathrm{~B}$ & 1 \\
\hline Enrofloxacin & $\mathrm{A}$ & 1 & $\mathrm{~A}$ & 2 & $\mathrm{~A}$ & 1 & $\mathrm{~A}$ & 1 & $\mathrm{~A}$ & 2 \\
\hline Thiabendazole & A, B & 4 & $\mathrm{~A}, \mathrm{~B}$ & 2 & $\mathrm{~A}, \mathrm{~B}$ & 2 & $\mathrm{~A}, \mathrm{~B}$ & 2 & A, B & 4 \\
\hline Sarafloxacin & - & - & A & 10 & $\mathrm{~A}$ & 2 & $\mathrm{~A}$ & 2 & - & - \\
\hline Sulfadimidine & A, B & 1 & $\mathrm{~A}, \mathrm{~B}$ & 1 & $\mathrm{~A}, \mathrm{~B}$ & 1 & $\mathrm{~A}, \mathrm{~B}$ & 1 & $\mathrm{~A}, \mathrm{~B}$ & 1 \\
\hline Sulfamonomethoxine & A, B & 1 & $\mathrm{~A}, \mathrm{~B}$ & 1 & $\mathrm{~A}, \mathrm{~B}$ & 1 & A, B & 1 & $\mathrm{~A}, \mathrm{~B}$ & 1 \\
\hline Pyrimethamine & A, B & 1 & B & 1 & B & 1 & A, B & 1 & $\mathrm{~A}, \mathrm{~B}$ & 1 \\
\hline Sulfamethoxazole & $\mathrm{A}, \mathrm{B}$ & 2 & B & 2 & $\mathrm{~A}, \mathrm{~B}$ & 2 & A, B & 2 & $\mathrm{~A}, \mathrm{~B}$ & 2 \\
\hline Florfenicol & $\mathrm{B}$ & 4 & A, B & 4 & B & 2 & A, B & 4 & $\mathrm{~A}, \mathrm{~B}$ & 4 \\
\hline Oxfendazole & $\mathrm{A}, \mathrm{B}$ & 1 & B & 1 & $\mathrm{~A}, \mathrm{~B}$ & 1 & $\mathrm{~A}, \mathrm{~B}$ & 1 & A, B & 1 \\
\hline Oxolinic acid & A, B & 2 & $\mathrm{~A}$ & 2 & $\mathrm{~A}$ & 1 & $\mathrm{~A}$ & 1 & $\mathrm{~A}$ & 2 \\
\hline Sulfaquinoxaline & $\mathrm{A}, \mathrm{B}$ & 2 & $\mathrm{~A}, \mathrm{~B}$ & 1 & A, B & 1 & $\mathrm{~A}, \mathrm{~B}$ & 1 & $\mathrm{~A}, \mathrm{~B}$ & 1 \\
\hline Sulfadimethoxine & $\mathrm{A}, \mathrm{B}$ & 1 & $\mathrm{~A}, \mathrm{~B}$ & 1 & $\mathrm{~A}, \mathrm{~B}$ & 1 & A, B & 1 & $\mathrm{~A}, \mathrm{~B}$ & 1 \\
\hline Oxfendazole sulfone & $\mathrm{A}, \mathrm{B}$ & 1 & $\mathrm{~A}, \mathrm{~B}$ & 1 & $\mathrm{~A}, \mathrm{~B}$ & 1 & $\mathrm{~A}, \mathrm{~B}$ & 1 & A & 1 \\
\hline Nalidixic acid & $\mathrm{A}, \mathrm{B}$ & 2 & $\mathrm{~A}$ & 2 & $\mathrm{~A}$ & 2 & A & 1 & $\mathrm{~A}$ & 2 \\
\hline Flubendazole & $\mathrm{A}, \mathrm{B}$ & 1 & $\mathrm{~B}$ & 1 & $\mathrm{~A}, \mathrm{~B}$ & 1 & $\mathrm{~A}, \mathrm{~B}$ & 1 & $\mathrm{~A}, \mathrm{~B}$ & 1 \\
\hline Fenbendazole & $\mathrm{A}, \mathrm{B}$ & 1 & $\mathrm{~B}$ & 1 & A, B & 1 & $\mathrm{~A}, \mathrm{~B}$ & 1 & A & 1 \\
\hline Nicarbazin & $\mathrm{A}, \mathrm{B}$ & 1 & $\mathrm{~A}, \mathrm{~B}$ & 1 & $\mathrm{~A}, \mathrm{~B}$ & 1 & $\mathrm{~A}, \mathrm{~B}$ & 1 & A, B & 1 \\
\hline Triclabendazole sulfoxide & $\mathrm{A}, \mathrm{B}$ & 4 & A & 4 & B & 2 & $\mathrm{~A}, \mathrm{~B}$ & 2 & $\mathrm{~A}, \mathrm{~B}$ & 4 \\
\hline Diclazuril & A, B & 4 & $\mathrm{~A}, \mathrm{~B}$ & 2 & $\mathrm{~A}$ & 2 & A, B & 2 & - & - \\
\hline Triclabendazole sulfone & $\mathrm{A}, \mathrm{B}$ & 1 & $\mathrm{~A}, \mathrm{~B}$ & 1 & $\mathrm{~A}$ & 1 & A, B & 1 & $\mathrm{~A}$ & 1 \\
\hline Triclabendazole & $\mathrm{A}, \mathrm{B}$ & 4 & - & - & $\mathrm{A}, \mathrm{B}$ & 4 & - & - & - & - \\
\hline Decoquinate & $\mathrm{A}, \mathrm{B}$ & 1 & B & 1 & $\mathrm{~A}, \mathrm{~B}$ & 1 & $\mathrm{~A}$ & 1 & B & 1 \\
\hline Eprinomectin & B & 2 & B & 2 & B & 4 & $\mathrm{~A}, \mathrm{~B}$ & 2 & B & 2 \\
\hline Closantel & $\mathrm{A}$ & 1 & A, B & 1 & $\mathrm{~A}, \mathrm{~B}$ & 4 & $\mathrm{~A}, \mathrm{~B}$ & 2 & $\mathrm{~A}, \mathrm{~B}$ & 1 \\
\hline Semduramicin & $\mathrm{A}, \mathrm{B}$ & 1 & $\mathrm{~A}, \mathrm{~B}$ & 1 & $\mathrm{~A}, \mathrm{~B}$ & 1 & $\mathrm{~A}, \mathrm{~B}$ & 1 & A, B & 1 \\
\hline Doramectin & B & 2 & B & 8 & $\mathrm{~A}, \mathrm{~B}$ & 4 & - & - & $\mathrm{A}, \mathrm{B}$ & 2 \\
\hline Lasalocid & A & 1 & - & - & B & 1 & B & 1 & B & 1 \\
\hline Moxidectin & $\mathrm{B}$ & 2 & $\mathrm{~B}$ & 1 & A, B & 1 & B & 1 & $\mathrm{~A}, \mathrm{~B}$ & 1 \\
\hline Ivermectin & B & 2 & B & 10 & B & 10 & $\mathrm{~A}, \mathrm{~B}$ & 10 & B & 10 \\
\hline Maduramicin & $\mathrm{A}, \mathrm{B}$ & 1 & $\mathrm{~A}, \mathrm{~B}$ & 1 & $\mathrm{~A}, \mathrm{~B}$ & 1 & B & 2 & A, B & 1 \\
\hline Salinomycin & B & 4 & B & 4 & B & 1 & $\mathrm{~A}, \mathrm{~B}$ & 1 & $\mathrm{~A}, \mathrm{~B}$ & 2 \\
\hline Monensin & A, B & 1 & $\mathrm{~A}, \mathrm{~B}$ & 1 & $\mathrm{~A}, \mathrm{~B}$ & 1 & A, B & 1 & $\mathrm{~A}, \mathrm{~B}$ & 1 \\
\hline Narasin & $\mathrm{A}, \mathrm{B}$ & 1 & $\mathrm{~A}, \mathrm{~B}$ & 1 & B & 1 & $\mathrm{~A}, \mathrm{~B}$ & 1 & $\mathrm{~A}, \mathrm{~B}$ & 1 \\
\hline
\end{tabular}

— Did not meet the criteria of the guideline with both methods, so LOQ was not evaluated.

\section{Quantification}

Calibration curves were obtained from the matrixmatched calibration samples. Blank samples were prepared as described above, and then matrix-matched standard solutions were prepared by mixing $900 \mu \mathrm{L}$ of blank solution and $100 \mu \mathrm{L}$ of an appropriately diluted working solution. The final concentrations of the matrixmatched standard solutions were 1, 2, 4, 10, 20, 25, 50, $100 \mu \mathrm{g} / \mathrm{L}$.

\section{Performance characteristics}

The accuracy of the method was evaluated according to the "Guidelines for the Validation of Analytical Methods for Residual Agricultural Chemicals in Food” notified by the Ministry of Health, Labour and Welfare in
Japan. The trueness, repeatability and within-laboratory reproducibility were determined by fortified recovery tests using two different concentrations (10 and $100 \mu \mathrm{g} /$ $\mathrm{kg}$ ), performed with two samples per day on five different days. The obtained results were evaluated by oneway analysis of variance. Selectivity was determined by analyzing blank samples. The limits of quantification (LOQs) were estimated by analyzing blank samples fortified at the levels of $0.25,0.5,1$ and $2.5 \mu \mathrm{g} / \mathrm{kg}$, and the lowest concentration of an analyte for which the signalto-noise ratio was over 10 was determined.

\section{Results and Discussion}

Analytical procedures, LC-MS/MS conditions and extraction processes basically followed those of previous 
studies ${ }^{11)}$. The target analytes were selected in accordance with the inspection items of Tokyo Metropolitan Institute of Public Health. However in the extraction process for milk analysis, the addition of water to the sample was skipped because the recovered amount of extraction solvent and the recoveries of each analyte were almost equal to those obtained when water was added.

Matrix effects were studied by comparing the peak areas of $25 \mu \mathrm{g} / \mathrm{L}$ matrix-matched standard solutions versus solvent standard solutions (Table 2). It had been considered that a large number of analytes could be quantified by the use of external calibration curves. However, matrix-matched calibration curves were adopted due to the fact that some analytes were greatly affected by matrices derived from food samples. Specifically, levamisole, ormethoprim, oxfendazole sulfone, 5-propylsulfonyl- $1 H$ benzimidazole-2-amine, pyrimethamine, sulfadimidine, sulfamerazine, sulfamonomethoxine, sulfaquinoxaline, thiabendazole and trimethoprim were only slightly affected by each matrix derived from food samples in both Method A and B. In contrast, fluoroquinolones (especially ciprofloxacin, danofloxacin and sarafloxacin) and macrolide antiparasites (especially doramectin, eprinomectin and moxidectin) were greatly affected and enhanced in both Methods A and B. Polyethers (especially maduramicin, salinomycin and semduramicin) tended to be suppressed by food matrices in Method A.

To validate the method, recovery tests using both Methods A and B were performed in accordance with Japanese guidelines, and the results are shown in Tables 3 and 4. Quantification values were obtained from matrix-matched calibration curves, where the correlation coefficient $\left(r^{2}\right)$ was over 0.991. In Method A, the number of analytes that satisfied the criteria of the guidelines was 33 out of 43 in milk, 25 in salmon, 30 in tiger shrimp, 36 in red sea bream and 32 in bastard halibut. In Method B, the results were 37 analytes in milk, 30 in salmon, 35 in tiger shrimp, 34 in red sea bream and 32 in bastard halibut. The pattern of recovery rates was similar to that in the previous study ${ }^{11)}$. Specifically, fluoroquinolones were not sufficiently recovered even when Method A was used, while polyethers and macrolides were well recovered using Method B. This indicates that this QuEChERS-based methodology is not suitable for analyzing amphoteric and highly polar compounds such as fluoroquinolones and antibiotics ${ }^{11,12)}$. This is because those compounds are much more soluble in water than acetonitrile, even though sodium chloride is used for salting out.

The selectivity of each analyte was confirmed by analyzing blank samples. There were no interfering peaks or peaks over one-third of MRL at the same retention times as the target analytes.

The estimated LOQs are shown in Table 5. The values are those of the methods that exhibited higher recoveries. They were lower than or equal to the MRL in each case.

\section{Conclusion}

In this study, the rapid analysis of multi-class residual veterinary drugs in milk, fish and shellfish was validated according to Japanese validation guidelines. Analytical procedures were performed using LC-MS/MS, and it took only $18 \mathrm{~min}$ to analyze 43 analytes. Sample preparation procedures were adopted from the QuEChERS methodology using two extraction solvents; Method A, which uses $1 \mathrm{vol} \%$ formic acid in acetonitrile, provided higher recoveries of quinolones, and Method B, which did not include acid, was used for polyethers and macrolides. Using these methods, the numbers of analytes that were validated in accordance with Japanese guidelines were 40 analytes out of 43 in milk, 37 in salmon, 42 in tiger shrimp, 41 in red sea bream and 39 in bastard halibut.

\section{References}

1) Wegener, H. C., Aarestrup, F. M., Gerner-Smidt, P., Bager, F. Transfer of antibiotic resistant bacteria from animals to man. Acta Vet. Scand. Suppl., 92, 51-56 (1999).

2) Noguchi, S., Terada, H., Tamura, Y. Simultaneous determination of veterinary drugs in livestock foods and seafoods using liquid chromatography/tandem mass spectrometry. J. Food Hyg. Soc. Japan, 49, 177-188 (2008).

3) Kajita, H., Hatakeyama, E. Simultaneous determination of residual veterinary drugs in livestock products and fish by liquid chromatography with tandem mass spectrometry. J. Food Hyg. Soc. Japan, 49, 381-389 (2008).

4) Frenich, A.G., Aguilera-Luiz, M.M., Martinez Vidal, J.L., Romero-Gonzalez, R. Comparison of several extraction techniques for multiclass analysis of veterinary drugs in eggs using ultra-high pressure liquid chromatographytandem mass spectrometry. Anal. Chim. Acta, 661, 150160 (2010).

5) Jimenez, V., Rubies, A., Centrich, F., Companyo, R., Guiteras, J. Development and validation of a amulticlass method for the analysis of antibiotic residues in eggs by liquid chromatography-tandem mass spectrometry. J. Chromatogr. A, 1218, 1443-1451 (2011).

6) Aguilera-Luiz, Martinez Vidal, J. L., M. M., Romero-Gonzalez, R., Frenich, A. G. Multiclass method for fast determination of veterinary drug residues in baby food by ultra-high-performance liquid chromatography-tandem mass spectrometry. Food Chem., 132, 2171-2180 (2012).

7) Chen, Y., Al-Taher, F., Juskelis, R., Wong, J. W., Zhang, K., Hayward, D. G., Zweigenbaum, J., Stevens, J. Multiresidue pesticide analysis of dried botanical dietary supplements using an automated dispersive SPE cleanup for QuEChERS and high-performance liquid chromatography-tandem mass spectrometry. J. Agric. Food Chem., 60, 9991-9999 (2012).

8) Ishii, R., Takahashi, K., Matsumoto, R. Analysis of imidocarb in livestock and seafood products using LC-MS/ MS. Food Hyg. Saf. Sci., 52, 34-39 (2011).

9) Kanda, M., Sasamoto, T., Takeba, K., Hayashi, H., Kusano, T., Matsushima, Y., Nakajima, T., Kanai, S., Takano, I. Rapid determination of fumagillin residue in honey by liquid chromatography-tandem mass spectrometry using the QuEChERS method. J. AOAC Int., 94, 878-885 (2011). 
10) Saito, S., Sakai, T., Nemoto, S., Matsuda, R. Determination of pindone in animal products, fishery products and honey by LC-MS/MS. Food Hyg. Saf. Sci., 52, 294-298 (2011).

11) Nakajima, T., Nagano, C., Sasamoto, T., Hayashi, H., Kanda, M., Kanai, S., Takeba, K., Matsushima, Y., Takano, I. Determination and validation of rapid analysis method for multi-class veterinary drugs in livestock products by LC-MS/MS. Food Hyg. Saf. Sci., 53, 243-253 (2012).

12) Nakajima, T., Sasamoto, T., Hayashi, H., Kanda, M., Takeba, K., Kanai, S., Kusano, T., Matsushima, Y., Takano, I. Screening assay of residual antibiotics in livestock samples by LC-MS/MS. Food Hyg. Saf. Sci., 53, 91-97 (2012). 